\title{
Flip-flop cycles in solar and stellar activity
}

\author{
Svetlana V. Berdyugina ${ }^{1,2}$ \\ ${ }^{1}$ Institute of Astronomy, ETH Zurich, CH-8092 Zurich, Switzerland \\ email: sveta@astro.phys.ethz.ch \\ ${ }^{2}$ Tuorla Observatory, University of Turku, Väisäläntie 20, FI-21500 Piikkiö, Finland
}

\begin{abstract}
We discuss flip-flop cycles in solar and stellar activity.
Keywords. Sun: activity, stars: activity, stars: late-type, stars: spots
\end{abstract}

Decades of continuous photometric monitoring of RSCVn-type stars (binaries with cool active giants or subgiants) revealed that large spots maintained their identities for years which was interpreted as a signature of one or two active longitudes. Berdyugina \& Tuominen (1998) showed that active longitudes on these stars are persistent structures which can however continuously migrate in the orbital reference frame. The active longitudes are separated by $180^{\circ}$ on average and differ in their activity level. Periodic switching of the dominant activity from one active longitude to the other results in a socalled flip-flop cycle. This was detected in both light curve variations and Doppler images (Fig. 1; Berdyugina \& Tuominen 1998; Berdyugina et al. 1998). Two active longitudes and flip-flops seem to be typical patterns of stellar activity. In addition to RS CVn stars they have been found on FK Com-type stars (Jetsu et al. 1993; Korhonen et al. 2002) and very active young solar analogues (Berdyugina \& Järvinen 2005).

Persistent active longitudes and a flip-flop cycle have also been found in the distribution of sunspots (Berdyugina \& Usoskin 2003). Large sunspot groups in both northern and southern hemispheres are preferably formed around two active longitudes which are separated by $180^{\circ}$ and persistent for at least 120 years. Similar to young solar-type dwarfs, the two active longitudes on the Sun are long-lived quasi-rigid structures which are not fixed in any reference frame due to differential rotation. They continuously migrate, with a variable rate, with respect to the Carrington system, especially in the beginning of the solar cycle (Fig. 2). The migration of active longitudes is caused by changes in the mean latitude of the sunspot formation and by differential rotation.

More than a dozen of active stars exhibiting flip-flop cycles enable a statistical analysis of their properties. There is a noticeable trend for stars with longer rotational periods to have longer flip-flop cycles (Fig. 3, left panel). The trend is prominent for RSCVn binaries as they have a wide range of rotation periods which are synchronized with their orbital motion. In connection to the spot activity cycle (analogous to the 11-yr sunspot cycle), there are clearly two groups of stars with cycle ratios 2:1 and 1:3 (Fig. 3, right panel). This implies different dominant dynamo mechanisms operating in these stars. The presence of binaries in both groups excludes possible effects of binarity on this ratio. However, it appears that the two groups greatly differ by the differential rotation rate, which might provide a clue to the nature of their dynamos.

\section{References}

Berdyugina, S. V., \& Järvinen, S. P. 2005, AN, 326, 283

Berdyugina, S. V., \& Tuominen, I. 1998, A\&A (Letters), 336, L25

Berdyugina, S. V., \& Usoskin, I. G. 2003, A\&A A, 405, 1121

Berdyugina, S.V., Berdyugin, A. V., Ilyin, I., \& Tuominen, I. 1998, A\& A, 340, 437

Jetsu, L., Pelt, J., \& Tuominen, I. 1993, A\&̈A, 278, 449

Korhonen, H., Berdyugina, S. V., \& Tuominen, I. 2002, A\& A, 390, 179 

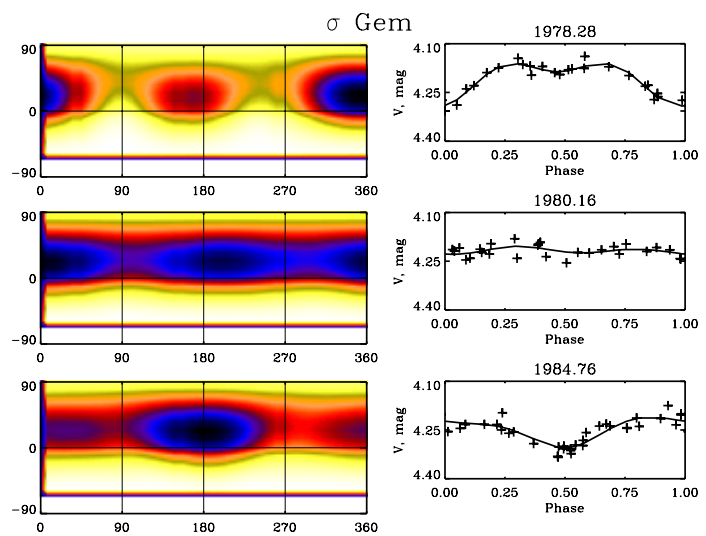

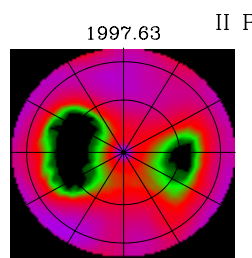

1998.54
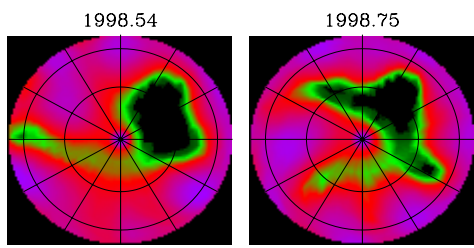

Figure 1. The flip-flop phenomenon on cool active binary components: on the left, as observed in light curves of $\sigma \mathrm{Gem}$, and on the right, in Doppler images of II Peg. The images on the left show the distribution of the spot filling factor on the stellar surface obtained via inversions of the light curves (plots in the middle). The II Peg images obtained from inversions of spectral line profiles show the temperature distribution on the stellar surface as seen from the pole. Flip-flops appear as a switch of the dominant activity to the opposite longitude.

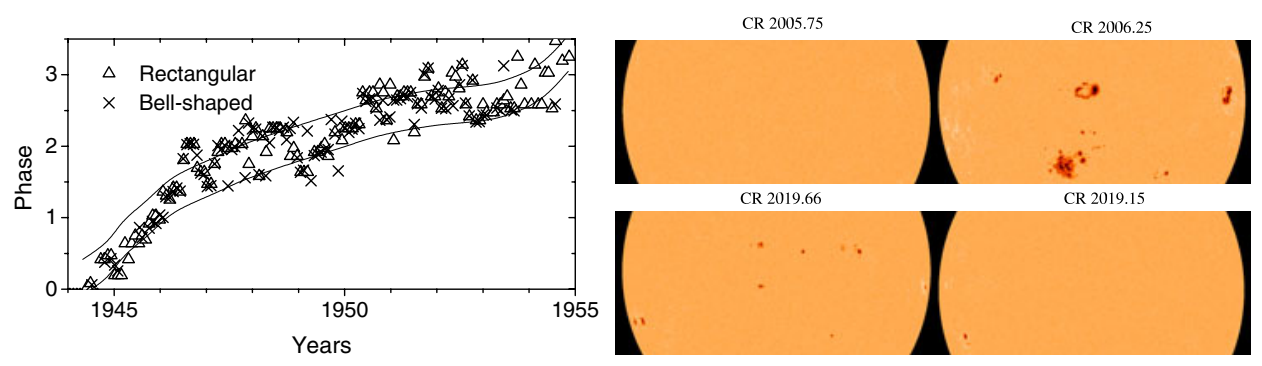

Figure 2. The flip-flop phenomenon on the Sun. On the left, rotational phases (in the Carrington system) of the biggest sunspot-cluster in the North are shown for cycle 18. The phases obtained with two different methods are denoted by different symbols (see Berdyugina \& Usoskin 2003). On the right, illustration of a flip-flop in cycle 23: two opposite sides of the Sun (SOHO-MDI, only equatorial region) are shown for two Carrington rotations (CR) one year apart. The maximum activity switched from the phase $\sim 0.25$ to the phase $\sim 0.66$.
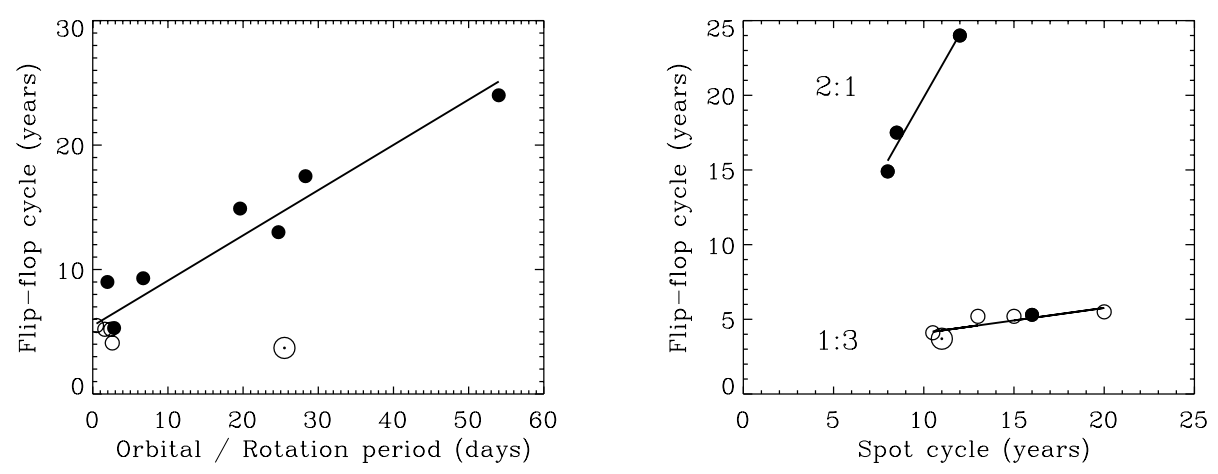

Figure 3. Flip-flop cycles on cool active stars: binaries (filled circles), single young stars (open circles), and the Sun (big circle with dot). On the left, correlation of the flip-flop cycles versus the orbital (binaries) or rotational (single stars) periods. On the right, the relation with the sunspot-like cycles are shown. Two groups with the cycle ratios of 1:3 and 2:1 are clearly seen. 